\title{
Designing Micromachined Accelerometers with Interferometric Detection
}

\author{
Maximillian Perez \\ Mechanical \& Aerospace Eng. \\ University of California, Irvine \\ Irvine, California 92697-3975 \\ Email: perezma@uci.edu \\ Telephone: (949) 824-6314
}

\author{
E. Jesper Eklund \\ Electrical Eng. \& Computer Science \\ University of California, Irvine \\ Irvine, California 92697-3975 \\ Email: eeklund@uci.edu
}

\author{
Andrei M. Shkel \\ Mechanical \& Aerospace Eng. \\ University of California, Irvine \\ Irvine, California 92697-3975 \\ Email: ashkel@uci.edu
}

\begin{abstract}
This paper explores designs for the implementation of high sensitivity accelerometers based on Fabry-Pérot Interferometers. Although such structures have the potential to achieve $\mu \mathrm{g}$ resolutions, design and implementation challenges can be limiting. This paper discusses the creation of such devices using two distinct proof mass and optical designs: one of a monolithic flexure with a thin film metallic reflector and another of an elastomeric flexure with a thin film multilayer dielectric reflector. Each device was fabricated, tested and characterized and conclusions about the advantages and disadvantages of the different design features are presented.
\end{abstract}

\section{INTRODUCTION}

The Fabry-Pérot Interferometer (FPI) is an optical device that can be constructed of two closely-spaced parallel plates with highly reflective inner surfaces. These plates create an optical resonance cavity which define a transmitted interferometric fringe. The spectral placement of the fringe is dependent on the spacing between the plates making devices suitable for a variety of sensor applications. However, nanometer tolerances in processing, construction and during operation are required for high-resolution performance.

Since the operation is power-passive and the signal is entirely optical, such sensors can be used in applications that require long-term or remote installation, are subject to electromagnetic interference or are in electrically sensitive environments. In addition, such devices could be integrated into existing optical communications networks for the physical monitoring of communications infrastructure.

An FPI-based accelerometer structure can be formed by using one plate as a proof-mass and the other as an inertial reference. The plane-normal deflection of proof-mass plate relative to the reference plate in the presence of an inertial load cause a shift in the spectral placing of the transmitted fringe (Fig. 2). For quasi-static operation, this is given by

$$
a=\frac{-\omega_{n}^{2} n \Delta \lambda}{2}
$$

where $\omega_{n}$ is the natural frequency of the proof-mass structure, $\Delta \lambda$ is the spectral shift from rest of the fringe which the device is operating about, and $n$ is the interferometric order of that fringe. It can be seen from this relationship that the sensitivity

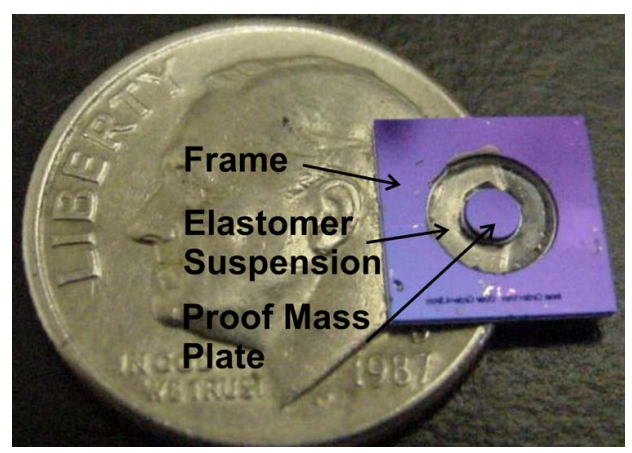

Fig. 1. Elastomer Suspended Proof Mass Structure.

and resolution of an FPI-based accelerometer is controlled by both its optical $(\Delta \lambda)$ and mechanical $\left(\omega_{n}\right)$ characteristics. Such devices have the potential for $\mu \mathrm{g}$ resolutions and could be serialized into extended sensor networks [1].

\section{FABRICATION}

Two fabrication approaches were explored in the construction of FPI-based accelerometers. Both incorporated deepreactive-ion-etching (DRIE) bulk micromachining of silicon wafers to take advantage of the higher mass and preferential surface quality (smoothness, flatness) of such processes over surface micromachining. One device was fabricated using timed-etch wafer thinning to construct the flexure mechanism along with a single layer metallic optical thin film reflective coating (Monolithic Suspension/Silver Coating). The other device was constructed using a combination of bulk micromachining and micromolding techniques to form a wafer-free flexure mechanism of an elastomeric polymer in combination with an optical multilayer thin film stack interference filter reflective coating (Elastomeric Suspension/Multilayer Coating) (Fig. 3,4).

The elastomeric device is fabricated by depositing on one side of a double sided polished wafer (DSP) two sets of quarter wave dielectric stacks (QWDS) of alternating PECVD (Plasma-Therm 790) deposited $\mathrm{SiO}_{2}$ and amorphous Si (aSi) films separated by a half wave stack of a-Si to form the high reflectance cavity surfaces of the device [2]. On the other 


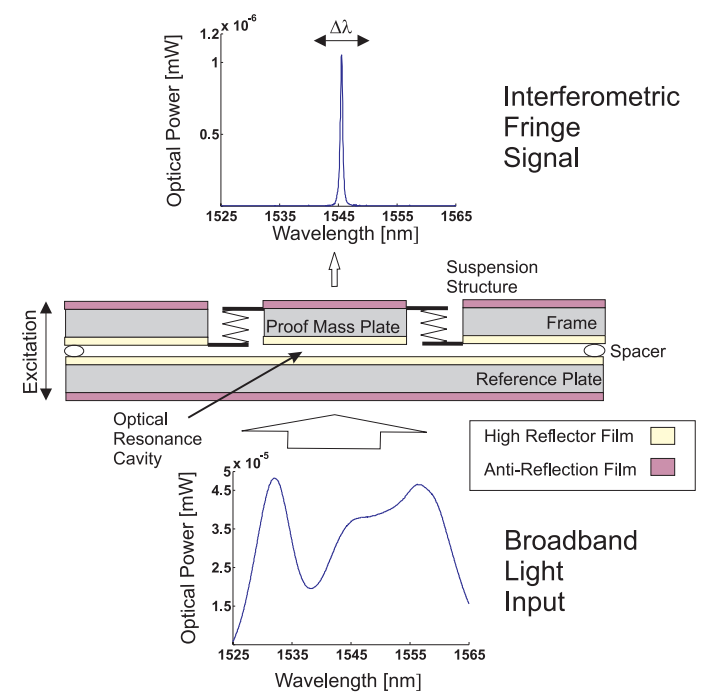

Fig. 2. Generalized Operation of the FPI-based Sensor.

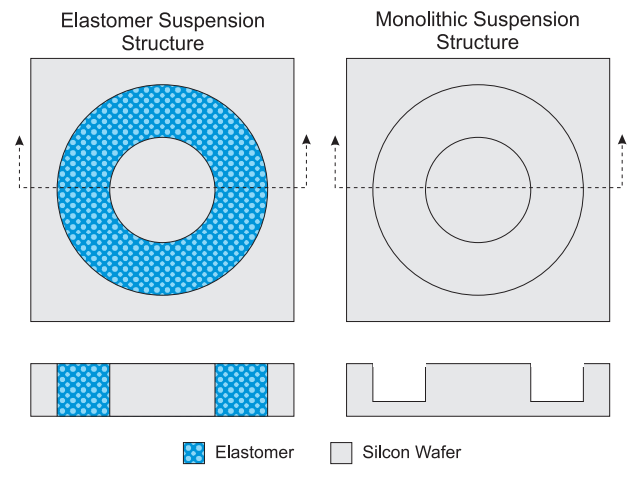

Fig. 3. Competing Proof Mass Structures

side, an anti-reflectance (AR) layer of stoichiometric silicon nitride $\left(S i_{x} N_{y}\right)$ is deposited. A thick photoresist (AZ4620) layer is spun on and is lithographically patterned. The wafer is attached to a handel wafer via a water soluble adhesive (Crystalbond 555) and a DRIE system (STS MESC ICP Etcher) is used to through-etch the wafer from the AR surface to the QWDS surface. The flexure suspension structures are formed while the device wafer is still attached to the handel wafer, by depositing catalyzed liquid polymer (Dow Corning Sylgard (c) 184) into the etched trench via a pipette using the through-etch channels as a mold. This polymer is cured at room temperature overnight forming a solid, but compliment elastomer providing a well defined flexure for the proof mass (Fig. 1). The devices are removed by melting the adhesive and dissolving any residual adhesive in warm water.

The fabrication and construction of the monlithic/silver device has been described previously and both devices are assembled in the process described in that work [3].

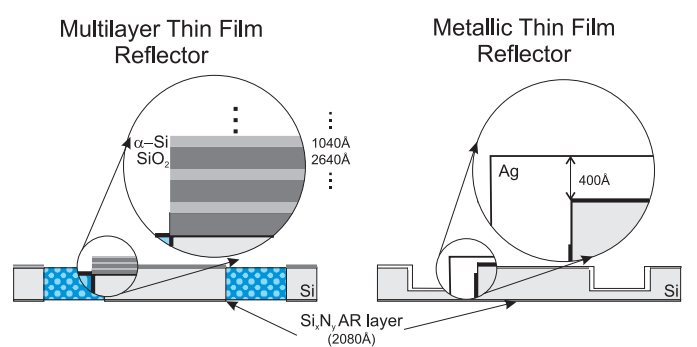

Fig. 4. Competing Reflector Structures

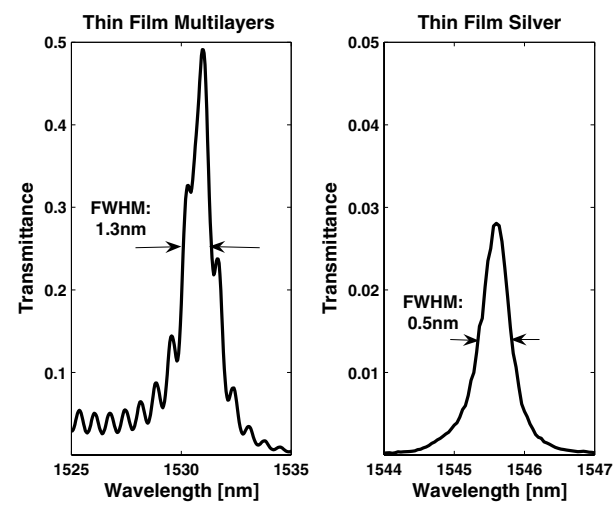

Fig. 5. FPI-based Sensors Optical Characteristics

\section{Characterization}

\section{A. Optical}

The optical quality of an FPI is given by its finesse (N), which is a measure of the ratio of spacing of the fringes to their width. The finesse of an FPI increases with plate reflectance and decreases with deviation of the plates from ideal parallel reflectors. In most sensing applications, the resolution to which $\Delta \lambda$ can be determined is related to the finesse.

Before testing, the two samples described previously are optically characterized. Using a broadband $(1525 \mathrm{~nm}<\lambda<$ $1565 \mathrm{~nm}$ ) light source (HP 83438A) and an optical spectrum analyzer (Agilent 86140B), the transmittance characteristics of the interferometric fringe is obtained (Fig. 5) and the full width at half maximum (FWHM) is derived. A tunable laser (HP 8168E) is used to obtain the free spectral range (FSR) of the interferometer by sweeping to determine the wavelength spacing between neighboring fringes. The optical finesse is calculated as the ratio between the two [4].

TABLE I

FPI SENSOR OPTICAL CHARACTERISTICS

\begin{tabular}{|c||c||c||c||c||c||c|}
\hline Coating & $\mathrm{n}$ & FWHM & FSR & $\mathrm{N}$ & $\frac{\delta T}{\delta \lambda} @-3 \mathrm{db}$ & $T_{\max }$ \\
\hline Silver & 53 & $0.5 \mathrm{~nm}$ & $30 \mathrm{~nm}$ & 60 & $0.083 \mathrm{~nm}^{-1}$ & $3 \%$ \\
\hline Multilayer & 32 & $1.3 \mathrm{~nm}$ & $48 \mathrm{~nm}$ & 37 & $0.71 \mathrm{~nm}^{-1}$ & $49 \%$ \\
\hline
\end{tabular}


TABLE II

Proof Mass Structure Mechanical Characteristics

\begin{tabular}{|c||c||c||c||c|}
\hline Construction & $f_{n 1}$ & $f_{n 2}$ & $\left(f_{n 2} / f_{n 1}\right)^{2}$ & $B W_{\max }$ \\
\hline Monolithic & $53.7 \mathrm{kHz}$ & $103 \mathrm{kHz}$ & 3.7 & $21.5 \mathrm{kHz}$ \\
\hline Elastomer & $3.10 \mathrm{kHz}$ & $5.42 \mathrm{kHz}$ & 3.1 & $1.24 \mathrm{kHz}$ \\
\hline
\end{tabular}

\section{B. Mechanical - Finite Element Analysis}

Finite element models were created with 3D elements using MSC.Patran for both devices to determine their principle natural frequencies. A radially symmetric mesh was used to model the flexure suspension element. This mesh is refined until changes in the fundamental natural frequency dropped below 1 percent. An value of E=2.5 MPa for Sylgard $(184$ is calculated from the published indentation hardness values [5], [6]. MSC.Nastran Sol 103 (Normal Modes) is used to calculate the two principle vibrational modes and their natural frequencies (Table II).

For both cases, the principle mode is responsible for ideal sense operation and is the out-of-plane normal deflection of the plate relative to the frame. The operational frequency bandwidth $\left(B W_{\max }\right)$ of the devices, where linear operation is expected and quasi-static assumptions hold, is up to $40 \%$ of the normal mode natural frequency [7]. The second mode is a trunnion or out-of-plane torsional mode that can cause the 'de-tuneing' of the FPI fringe resulting in a lowering of finesse and reduction of the peak transmission. The value $\left(f_{n 2} / f_{n 1}\right)^{2}$ is equal to the quasi-static excited amplitude ratio between the $1^{\text {st }}$ and $2^{\text {nd }}$ modes when both are under unit excitation. This suggests that the structures are suited to responding principally in the first mode while rejecting undesirable crossaxis excitations of the second mode.

\section{DEMONSTRATION}

The devices are dynamically characterized by directing a tunable laser (HP 8168E) through each device and monitoring the transmitted power via an amplified photodetector (Thorlabs PDA255). The laser output power is fixed $(1000 \mu \mathrm{W})$ at the maximum transmission wavelength at rest for both devices. A reference accelerometer (Dytran 3030A) is fixed to an aluminum block along with the sample and aligned with the samples sensitive axis. Both the sample and the reference are excited via a manual shock on the platform on which the block was fixed (Fig. 6). As the interference fringe moves from its rest position under the inertial load, the transmitted optical power is attenuated according to the sample's optical and mechanical characteristics. Acceleration magnitude is registered as reductions in the transmitted optical power (Figs. 7 and 8).

By comparing the optical response of the sample and the response of the reference to initial excitation, the sensitivity of the device is estimated. The optical response of the FPI sensor is compared to reference response at the point of the initial shock impulse (Fig. 9). In this mode, the response of



Fig. 6. Test and Demonstration Platform


Fig. 7. Elastomer/Interferenece FPI Accelerometer Time Response

the device can be expressed as

$$
a=\frac{\omega_{n}^{2} n \Delta V}{2 P L G \frac{\delta T}{\delta \lambda}}
$$

where $\mathrm{P}$ is the optical power of the source, $\mathrm{L}$ are the fractional optical coupling losses not due to the FPI, G is the optical power to voltage gain (for PDA255 $G=9500 \mathrm{~V} / \mathrm{W}$ at $\lambda=1500 \mathrm{~nm}$ ), and $\frac{\delta T}{\delta \lambda}$ is slope of the fringe during operation.

\section{Discussion}

\section{A. Optical}

The materials used in the multilayer device are characterized by their low coefficient of extinction (' $k$ ') in the near IR wavelengths. All reflective metallic optical coatings are characterized by their high $\mathrm{k}$ values since $\mathrm{k}$ contributes to the coatings overall reflectivity. Since $\mathrm{k}$ determines the absorbtion losses in a material, this effect accounts for the large difference between the peak transmissivity of the devices with the multilayer device demonstrating a 10X improvement in optical signal-to-noise ratio. 

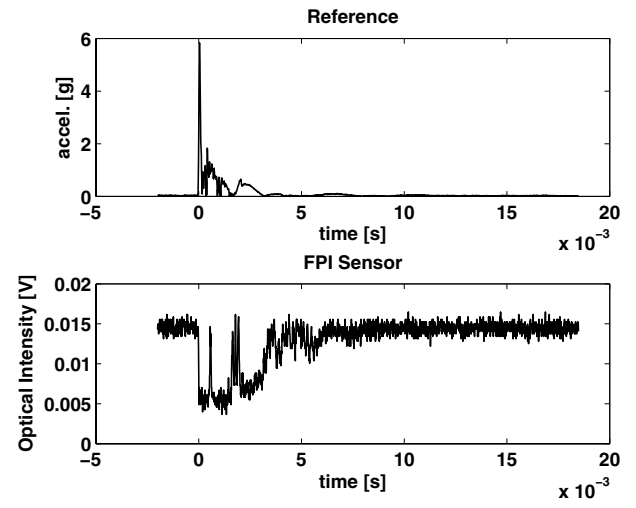

Fig. 8. Monolithic/Silvered FPI Accelerometer Time Response
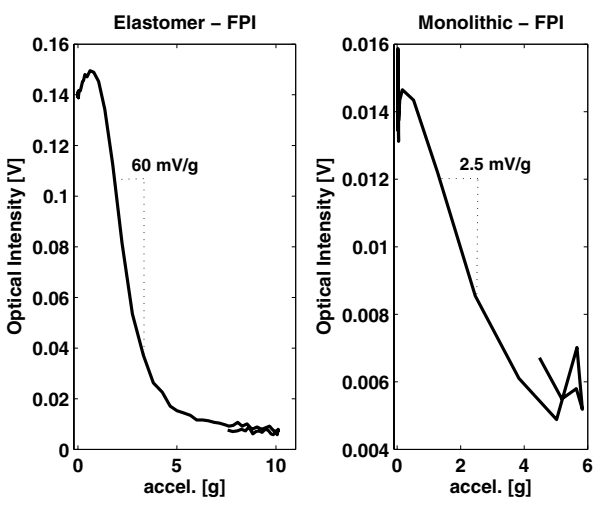

Fig. 9. FPI Sensors Response during initial shock.

Additionally, the optical losses limit the optical sensitivity of the metallic device. Despite the higher finesse of the silver film device compared to the multilayer device, its observed $-3 \mathrm{db}$ slope is less resulting in its lower optical sensitivity $\left(\frac{\delta T}{\delta \lambda}\right.$, Table I) limiting the final sensitivity of the metallic device [8].

In contrast to metallic thin films, thin film multilayer film reflectance is also dependant on design. In this case, the multilayer design was tuned towards the creation of serialized sensors and not to maximize finesse [9].

\section{B. Sensor Operation}

The agreement between the expected device sensitivities and those observed (Table III) is poor. The increase in observed sensitivity over that expected in the monolithic structure is thought to be due to the high stiffness of the flexure allowing other compliment elements, such as the spacers, to dominate the response of the assembled sensor.

TABLE III

FPI-BASED SENSOR CHARACTERISTICS

\begin{tabular}{|c||c||c|}
\hline Device & Sensitivity (Fig. 9) & Sensitivity (Eqn. 2) \\
\hline Monolithic/Silver & $2.5 \mathrm{mV} / \mathrm{g}$ & $0.13 \mathrm{mV} / \mathrm{g}$ \\
\hline Elastomer/Multilayer & $60 \mathrm{mV} / \mathrm{g}$ & $327 \mathrm{mV} / \mathrm{g}$ \\
\hline
\end{tabular}

The reduction in sensitivity over that expected in the elastomeric device is due to the relatively low 2 nd mode (trunnion) natural frequencies. Trunnion mode deflection will cause both a broadening and reduction in amplitude of the interferometric fringe during operation resulting in a decrease in overall device sensitivity [10]. Trunnion mode deflection is activated by coupling to the sense mode or through cross-axis rotational accelerations. Elastomeric structures could reduce these effects through the formation of aligned composites [11].

\section{CONCLUSION}

Two distinct accelerometer structures based on interferometric detection were developed, characterized, and demonstrated. The optical and mechanical features were independently evaluated and used to predict the device sensitivity. Both devices were operated as accelerometers and their sensitivities were characterized. Agreement exists between the predicted and observed sensitivities indicating expected operation but the differences suggest additional unmodeled dynamics. It is observed that low-loss reflectors, such as dieletectic multilayers, are needed for high sensitivity sensors and that such coatings have the potential for high resolution sensing, as well as allowing the extension of such sensors to systems of linear arrays. Elastomeric structures are demonstrated as an advantageous alternative fabrication technology by offering the flexibility to reduce cross-axis effects.

\section{ACKNOWLEDGMENT}

Supported in part by the National Science Foundation Grant CMS-0223050 and by UC Discovery/VIP Sensors grant no 9442531-19919-8 and NSF grant no CMS-0330470.

\section{REFERENCES}

[1] M. Perez, "Micromachined elastomeric accelerometers with embedded optical detection for serial sensor networks," Master's thesis, University of California, Irvine, 2004.

[2] H. A. Macleod, Thin-Film Optical Filters, 3rd ed. Institute of Physics Publishing, Bristol-Philadelphia, 2001.

[3] E. J. Eklund and A. M. Shkel, "Factors affecting the performance of micromachined sensors based on fabryperot interferometry," Journal of Micromechanics and Microengineering, vol. 15, pp. 1770-1776, July 2005.

[4] P. D. Atherton, N. K. Reay, J. Ring, and T. R. Hicks, "Tunable fabryperot filters," Optical Engineering, vol. 20, no. 6, pp. 806-814, 1981.

[5] D. Corning, "Information about high technology silicone materials," available from Dow Corning Corp.

[6] A. N. Gent, "On the relations between indention hardness and young's modulus," Transactions and proceedings (Institution of the Rubber Industry), vol. 34, no. 2, pp. 46-57, 1958.

[7] T. G. Beckwith, N. L. Buck, and R. D. Marangoni, Mechanical Measurements. Addison-Wesley, 1982.

[8] M. Born and E. Wolf, Princples of Optics, 7th ed. Pergamon, London, 1999.

[9] M. A. Perez and A. M. Shkel, "Conceptual design and preliminary characterization of serial array system of high-resolution mems accelerometers with embedded optical detection," Smart Structures and Systems, vol. 1, no. 1, pp. 63-82, January 2005.

[10] J. Moon and A. M. Shkel, "Analysis of imperfections in a micromachined tunable-cavity interferometer," SPIE Annual International Symposium on Smart Structures and Materials, March 2001.

[11] M. Perez and A. Shkel, "Elastomeric composites to reduce the effects of trunnion mode in inertial devices," NSTI Nanotech 2005 Conference, pp. 557-560, May 2005. 